\title{
Evaluation of Quinolone-Resistant Strains of Klebsiella pneumoniae in Clinical Specimens Obtained From Patients Referred to Zahedan Educational Hospitals
}

\author{
Shahram Shahraki ${ }^{1, ;}$; Farzaneh Mohammadzadeh-Rostami ${ }^{2}$; Mehdi Haddadi-Feishani ${ }^{2}$; \\ Amir Hossein Mohagheghifard ${ }^{3}$; Somayeh Jahani ${ }^{1}$; Hamidreza Majidiani ${ }^{4}$ \\ ${ }_{1}^{1}$ Infectious Diseases and Tropical Medicine Research Center, Zahedan University of Medical Sciences, Zahedan, IR Iran \\ ${ }_{3}^{2}$ Department of Microbiology, Zahedan University of Medical Sciences, Zahedan, IR Iran \\ ${ }^{3}$ Department of Virology, Zahedan University of Medical Sciences, Zahedan, IR Iran \\ ${ }^{4}$ Candidate of Veterinary Medicine, Faculty of Veterinary, Zabol University, Zabol, IR Iran \\ ${ }^{*}$ Corresponding author: Shahram Shahraki, Infectious Diseases and Tropical Medicine Research Center, Zahedan University of Medical Sciences, Zahedan, IR Iran. \\ E-mail: f.m.rostami@zaums.ac.ir
}

Received: February 4, 2014; Accepted: March 26, 2014

\begin{abstract}
Background: Klebsiella pneumoniae is among the most common and significant agents of community and hospital-acquired infections. Plasmid-mediated quinolone resistance (PMQR) was increasingly identified in Enterobacteriaceae family worldwide. Quinolones are broad-spectrum antibiotics the resistant to which has increasingly been reported among many bacterial species including Klebsiella. The current study was conducted to evaluate the prevalence of quinolone-resistant strains of K. pneumoniae in Zahedan during 2013 and 2014. Materials and Methods: In this cross-sectional study, 184 samples of $K$. pneumoniae were collected. Isolates were screened for quinolone antibiotics resistance using disk diffusion method according to clinical and laboratory standards institute (CLSI) guidelines. Also MIC (minimum inhibitory concentration) was used against the ciprofloxacin antibiotic by the dilution method in tubes.

Results: Based on the obtained results by the Agar disk diffusion test, $31.5 \%, 18.4 \%, 17.3 \%, 4.3 \%, 3.2 \%$ and $2.1 \%$ of the strains were resistant to nalidixic acid, ciprofloxacin, norfloxacin, ofloxacin, levofloxacin, and getifloxacin respectively.

Conclusions: Our results show increased prevalence of quinolone resistance among $K$. pneumoniae in south-east of Iran. This may stem from their irrational prescription thus it is recommended that their prescription be based on antibiotic sensitivity test results. We also recommend further evaluation using molecular techniques and also preventive measures.
\end{abstract}

Keywords: Quinolones; Disk diffusion; Klebsiella pneumoniae

\section{Background}

Klebsiella are oppotunitistic pathogens from the enterobacteriaciae family of bacteria which can cause neonatal enteritis, meningitis, urinary tract infections, soft tissue infections, bacteremia, and septicemia [1].

Klebsiella pneumoniae is one of the common causes of hospital acquired infections [2] additionally it is among the important agents of community-acquired infections [3]. Antibacterial resistance has always been a potentially major threat to the human health [4]. Thus, the world health organization nominated the year 2011 as "Antibiotic resistance". In addition to this, it has made recommendations to the governments on evaluating the antibiotic resistance status, appropriate antibiotic utilization, prescription-restricted antibiotic sale, and infection prevention and control in order to restrain and prevent the immergence of antibiotic resistance [5]. The continuous use of antibiotics and the resulting difficulty in antibiotic choice has led to an increasing rate of antibacterial resistance among Gram negative bacteria especially K. pneumoniae [6].

Quinolones inhibit DNA topoisomerases II and IV which play an important role in bacterial duplication. The main target of quinolones in Gram negative bacteria is the A subunit of DNA topoisomerase II while quinolones exert their effect on Gram positive bacteria via inhibiting DNA topoisomerase IV $[7,8]$. Variation in the two central rings permits the production of new quinolones under the name of fluoroquinolones $[9,10]$. In the current study we focused on assessing the prevalence of K. pneumoniae resistance to quinolones. In Iran, due to arbitrary drug overuse by patients, drug resistance has been increased severely and uncontrollably $[7,11]$ and there is not any information about resistance of quinolones antibiotics in the region. With consideration to

Copyright (C) 2015, Zahedan University of Medical Sciences. This is an open-access article distributed under the terms of the Creative Commons Attribution-NonCommercial 4.0 International License (http://creativecommons.org/licenses/by-nc/4.0/) which permits copy and redistribute the material just in noncommercial usages, provided the original work is properly cited. 
the prevalence of resistant bacteria, lack of necessary data in region and the importance of resistance organisms to fluoroquinolones, paying attention seems to be imperative.

The aim of this study was to evaluate the rate of resistance to quinolones in K. pneumoniae among clinical specimens obtained from patients referring to teaching hospitals of Zahedan, south-east of Iran in 2013 - 2014 to aid in controlling the antibiotic resistance state and in appropriate antibiotic choice by the physicians.

\section{Materials and Methods}

\subsection{Bacterial Isolation}

In this cross-sectional study, a total number of 184 specimens of urine, respiratory tract secretions, blood, ulcer and tracheal lavage, fluid on urinary catheters, joint effusion, and phlegm collected during 2013 and 2014 from patients referring to Khatam-al-Anbia, Bou-Ali, and Imam Ali hospitals in Zahedan, south-east of Iran. Urine was the most common among specimens $(132=71.7 \%)$ while there were two joint effusion fluid specimens and one phlegm specimen accounted for the lowest numbers among our samples. One hundred thirty six samples provided by Khatam-al-Anbia hospital, 30 samples from Imam Ali and 18 samples from Bou-Ali hospitals respectively.

All specimens were cultured (isolation technique) on blood agar and MacConkey agar selective medium. The colonies were then morphologically evaluated and studied using biochemical tests and conventional method such as DNase and other important method for isolation. (All culture media and reagents were purchased from Merck co. Germany) and finally identified by way of standard tables. Strains of K. pneumoniae were then isolated for use in the next stages of the project.

\subsection{Bacterial Plasmid DNA Extraction}

Bacterial plasmid DNA was conducted according to AccuPrep (Bioneer, South Korea) plasmid nano-plus plasmid mini extraction kit(Cat. No.: K-3112) protocols. The kit contained 6 buffers (named 1-5 and D), RNase A powder, and DNA extraction columns. The quantity of DNA was measured by spectrophotometry after extraction. For quality control, $3 \mu \mathrm{L}$ of DNA was electrophoresed on $2 \%$ agarose gel.

\subsection{Antimicrobial Susceptibility Testing}

Bacterial sensitivity was examined to quinolone antibiotics (nalidixic acid, ciprofloxacin, norfloxacin, ofloxacin, levofloxacin, and getifloxacin) with disk diffusion technique. Disks were ordered from MAST co. England. Standard strains of E. coli ATCC25922, and K. pneumoniae ATCC700603 were used for antibiogram quality control [12]. Minimum inhibitory concentration (MIC) was calculated for ciprofloxacin according to CLSI guideline [13].
Prior to the test, the Mueller-Hinton culture media were incubated at $37^{\circ} \mathrm{C}$ for 24 hours to rule out the possibility of contamination. Antibiotic disks were kept out of the refrigerator to accommodate to room temperature half an hour prior to the test. The plates were incubated at $37^{\circ} \mathrm{C}$ following bacterial culture and disk insertion. The zone of growth inhibition of each disk was measured with a ruler and results were recorded in millimeters [13]. Micro dilution broth test was used to calculate MIC for sensitivity of isolates to ciprofloxacin.

\section{Results}

In this study, 184 non-repetitive clinical samples including 132 urine samples (71.1\%), 32 respiratory excretions samples (17.3\%), 9 blood samples (4.8\%), 3 wound samples (1.6\%), 3 tracheal lavage (1.6\%), 3 samples of urinary catheter secretions (1.6\%), a sample of synovial fluid $(0.5 \%)$ and a sample of sputum (0.5\%) that were collected during one year from Khatam-al-Anbia, Imam Ali and Bou-Ali hospitals of Zahedan, went under evaluation and finally 184 strain of K. pneumoniae were acquired. Among these, 136 samples (73.9\%) were from Khatam-al-Anbia hospital, 30 samples (16.3\%) were from Imam Ali hospital and 18 samples (9.7\%) were from Bou-Ali hospital.

One hundred three patients (55.9\%) were female and 81 patients (44.2\%) were male and the average age for males was $27.5 \pm 18.2$ years and for females was $24.8 \pm 16$ years.

About disk diffusion in agar method findings, this method was done using 184 strain of K. pneumoniae isolated from clinical samples, according to the proposed methods of CLSI for the evaluation of nalidixic acid, ciprofloxacin, norfloxacin, ofloxacin, levofloxacin and gatifloxacin antibiotics. The result of disk agar diffusion has been shown in Table 1.

Thirty four isolates were resistant to ciprofloxacin $(\mathrm{MIC}=4)$. The results of antibiotic sensitivity testing with agar disk diffusion and MIC show that both methods to have equal results with no statistically significant difference in sensitivity as shown by Z-test with 95\% confidence. The statistical analysis was performed using SPSS-18.

Table 1. Antibiotic Resistance Patterns of Isolates to Antibiotics Used $^{\mathrm{a}, \mathrm{b}}$

\begin{tabular}{lccc}
\hline Antibiotics & S & I & R \\
\hline Nalidixic acid & $98(53.2)$ & $28(15.2)$ & $58(31.5)$ \\
\hline Ciprofloxacin & $103(55.9)$ & $47(25.5)$ & $34(18.4)$ \\
Norfloxacin & $114(61.9)$ & $38(20.6)$ & $32(17.3)$ \\
\hline Ofloxacin & $159(86.4)$ & $17(9.2)$ & $8(4.3)$ \\
\hline Levofloxacin & $161(87.5)$ & $17(9.2)$ & $6(3.2)$ \\
\hline Gatifloxacin & $165(89.6)$ & $15(8.1)$ & $4(2.1)$ \\
\hline
\end{tabular}

a Data are presented as No. (\%)

b S:Sensitive, I:Intermediate, R:Resistant. 


\section{Discussion}

In our study the highest antibiotic resistance occurred to nalidixic acid and the lowest was to levofloxacin and gatifloxacin. Quinolones and fluoroquinolones are a group of synthetic antibacterial drugs which nowadays are widely being used to treat the bacterial infections. Nalidixic acid, the first member of the group quinolones was discovered in 1962 [7]. Placing fluorine atom in position 6 was the first modification in the basic structure of quinolones that improved their antibacterial power and caused quinolones to be used as beneficial drugs treating respiratory and urinary systemic infections [9].

In this study, the resistance to nalidixic acid and ciprofloxacin antibiotics has been achieved 31.5\% and $18.4 \%$, respectively, that shows a progressive resistance. With regards to this fact that it has passed 6 decades since the production of nalidixic acid in 1962 as the first drug of quinolones and because it was used from the beginning for the treatment of specific urinary infections, so it expects that the resistance to the nalidixic acid be much more than the other quinolone antibiotics [3]. In a research done by Soltan-Dalal et al. [11] to determine the antibiotic resistance pattern of isolated Klebsiella species from patients samples in Imam Khomeini hospital in Tehran, the resistance to nalidixic acid was reported $2 \%$ and comparison with our results shows that the resistance to nalidixic acid is much higher in this study that could be due to poor hygiene and over usage of antibiotics in the region. In another study by Langarizadeh et al. [14] in Tabriz, the resistance rate to nalidixic acid 58.3\% and to ciprofloxacin and norfloxacin $43 \%$ have been reported that had accordance with results of this study which could be due to indiscriminate use and without accurate surveillance of these drugs in developing countries. Norfloxacin and ofloxacin are chemical drugs from the second age of quinolones that has been reported $17.3 \%$ and $4.3 \%$ respectively which in the case of norfloxacin has been lesser rather to the results of similar studies implemented by Madani et al. in Kermanshah [15], Molaabaszadeh et al. in Tabriz [16] and Fernandez in Spain [17] that reported the resistance rate 31.3\%, 29\% and 33\%, respectively. In a study which Rastegar Lari et al. [18] devoted to the Gram negative bacteria isolated from patients with urinary tracts infections, they reported the resistance rate to ofloxacin $13.27 \%$. Incidence of resistant isolates to ofloxacin in Japan has been reached from 13.5\% in 1995 to $32.5 \%$ in 1999. Resistance rate to the second age quinolones has been reported lesser in contrast to the first age ones in other regions which indicate that norfloxacin has not been administered as health priorities by physicians. Another second age fluoroquinolones is levofloxacin with Tavenax trade name which its resistance has been reported in 6 cases (3.2\%).

In Karlowsky et al. [19] research, during 1998 to 2001 they evaluated antibiotic resistance in Enterobacteriacea family isolated from conferring patients to the US hospitals and the resistance rate to K. pneumoniae, E. coli, Entero- bacter cloacae, Enterobacter aerogenes, Serratia marcescens, Morganella morgani, Proteus mirabilis and Providencia species has been reported 5.1\%, 7.4\%, 8.3\%, 4.4\%, 4.9\%, 20.5\%, $14.4 \%$ and $48.3 \%$, respectively. So with regards to appropriate condition in K. pneumoniae and progressive resistance in other family members, since the horizontal transition of resistance genes is probable, it seeks surveillance and accuracy in prescription of infection with other bacteria. Gatifloxacin is a new fluoroquinolones (fourth age) with stronger antibacterial effects against bacteria than the older fluoroquinolones. In this study the least resistance rate has been reported 4 cases (2.1\%).

In a study, Sader et al. [20] analyzed the global patterns of resistance of 21 antimicrobial agents against 48440 Enterobacteriacea during 1997 - 2001 in four international areas (Asia, Pacific ocean, Europe, Latin America and North America) and reported the resistance to gatifloxacin $5.9 \%$. Lower resistance to gatifloxacin in the region could be due to unavailability of the mentioned antibiotic in this area. The new age of quinolones, on the one hand, must conquest to multidrug resistances and on the other hand has lesser side effects. Although, dysglycemia, as its side effects, caused this drug to be collected from American markets, so effort for synthetizing gatifloxacin derivations with higher effects and more tolerance is necessary to remove the limitations of gatifloxacin. Irregular utility of antibiotics and also their administration without doing antibiotic susceptibility determination tests or high dose prescription of antibiotics are among the most important reasons for bacteria resistance. On the other hand, utilizing of wrong antibiotic also is one of the proofs for antibiotic resistance. The results of this study could be a guide for native physicians for aberrant administration of these efficient antibiotics with low side effects and the necessity of much more surveys with aid of molecular techniques from the point of absolute evaluation of resistance genetic pattern shows existence of accurate supervision and surveillance programs and rigid precautionary proceeding.

\section{Acknowledgements}

This paper has been extracted from project No. $6072 \&$ 6111 (Performer: Shahram Shahraki).

\section{Authors' Contributions}

All authors had equal role in design, work, statistical analysis and manuscript writing.

\section{Funding/Support}

Zahedan University of Medical Sciences, Zahedan, IR Iran.

\section{References}

1. Babypadmini S, Appalaraju B. Extended spectrum -lactamases in urinary isolates of Escherichia coli and Klebsiella pneumoniae prevalence and susceptibility pattern in a tertiary care hospital. Indian J Med Microbiol. 2004;22(3):172-4. 
2. Tsai YK, Fung CP, Lin JC, Chen JH, Chang FY, Chen TL, et al. Klebsiella pneumoniae outer membrane porins OmpK35 and OmpK36 play roles in both antimicrobial resistance and virulence. Antimicrob Agents Chemother. 2011;55(4):1485-93.

3. Luo Y, Yang J, Zhang Y, Ye L, Wang L, Guo L. Prevalence of beta-lactamases and 16S rRNA methylase genes amongst clinical Klebsiella pneumoniae isolates carrying plasmid-mediated quinolone resistance determinants. Int J Antimicrob Agents. 2011;37(4):352-5.

4. Green VL, Verma A, Owens RJ, Phillips SE, Carr SB. Structure of New Delhi metallo-beta-lactamase 1(NDM-1). Acta Crystallogr Sect F Struct Biol Cryst Commun. 2011;67(Pt 10):1160-4.

5. Lye DC, Kwa AL, Chlebicki P. World health day 2011: antimicrobial resistance and practical solutions. Ann Acad Med Singapore. 2011;40(4):156-7.

6. Mustafa OA, Yusuf D. Investigation of some antibiotic susceptibility plasmid profiles and ESBL characteristic of Klebsiella pneumoniae isolated from urinary system infection. World Appl Sci J. 2009;6(5):630-6.

7. Soleimani-Asl Y, Zibaei M, Firoozeh F. [Detection of qnrA gene among quinolone-resistant Escherichia coli isolated from urinary tract infections in Khorram Abad during 2011-2012]. Feyz. 2013;17(5):488-94.

8. Wang A, Yang Y, Lu Q, Wang Y, Chen Y, Deng L, et al. Presence of qnr gene in Escherichia coli and Klebsiella pneumoniae resistant to ciprofloxacin isolated from pediatric patients in China. BMC Infect Dis. 2008;8:68.

9. Nordmann P, Poirel L. Emergence of plasmid-mediated resis tance to quinolones in Enterobacteriaceae. J Antimicrob Chemother. 2005;56(3):463-9.

10. Tran JH, Jacoby GA, Hooper DC. Interaction of the plasmid-encoded quinolone resistance protein Qnr with Escherichia coli DNA gyrase. Antimicrob Agents Chemother. 2005;49(1):118-25.

11. Soltan-Dalal MM, Miremadi SA, Sharify-Yazdi MK. [Antimicrobial resistance trends of Klebsiella Spp. isolated from patients in
Imam Khomeini hospital] . Payavard Salamat. 2012;6(4):275-81.

12. Stephenson S, Brown PD, Holness A, Wilks M. The emergence of qnr-mediated quinolone resistance among Enterobacteriaceae in Jamaica. West Indian Med J. 2010;59(3):241-4.

13. Clinical and Laboratory Standards Institute. Performance standards for antimicrobial susceptibility testing: Twenty-first Informational Supplement M100-S21.PA, USA: CLSI, Wayne; 2011.

14. Langarizadeh N, Ahangarzadeh-Rezai M, Aghazadeh M, Hasani A [The prevalence of multi-drug resistant Klebsiella pneumoniae in children and adults with UTI referring to Tabriz health centers]. Oloum Zisti. 2010;4(1):9-17.

15. Madani H, Khazaee S, Kanani M, Shahi M. [Antibiotic resistance pattern of E. coli isolated from urine culture in Imam Reza hospital Kermanshah-2006]. Behbood J. 2008;12(3):287-95.

16. Molaabaszadeh H, Hajisheikhzadeh B, Mollazadeh M. Study of sensibility and antimicrobial resistance in Escherichia coli isolated from urinary tract infection in Tabriz city. J Fasa Univ Med Sci. 2013;3(2):149-54.

17. Fernandez A, Pereira MJ, Suarez JM, Poza M, Trevino M, Villalon P et al. Emergence in Spain of a multidrug-resistant Enterobacter cloacae clinical isolate producing SFO-1 extended-spectrum betalactamase. J Clin Microbiol. 2011;49(3):822-8.

18. Rastegar Lari A, Alaghehbandan R. Nosocomial infections in an Iranian burn care center. Burns. 2000;26(8):737-40.

19. Karlowsky JA, Kelly LJ, Thornsberry C, Jones ME, Sahm DF. Trends in antimicrobial resistance among urinary tract infection isolates of Escherichia coli from female outpatients in the United States. Antimicrob Agents Chemother. 2002;46(8):2540-5.

20. Sader HS, Jones RN, Gales AC, Winokur P, Kugler KC, Pfaller MA, et al. Antimicrobial susceptibility patterns for pathogens isolated from patients in Latin American medical centers with a diagnosis of pneumonia: analysis of results from the SENTRY Antimicrobial Surveillance Program (1997). Diagn Microbiol Infect Dis. 1998;32(4):289-301. 